\title{
Delay Analysis for a Reliable Message Delivery in Sparse Vehicular Ad Hoc Networks
}

\author{
Atef Abdrabou§̧, Ben Liang $\dagger$, and Weihua Zhuang $\ddagger$ \\ Email:\{atef.abdrabou@uaeu.ac.ae, liang@comm.utoronto.ca, wzhuang@bbcr.uwaterloo.ca\}
}

\begin{abstract}
In this paper, we address the relation between message delivery delay and reliability for the communication between a vehicle and a road side unit (RSU). We focus on sparse or low density vehicular ad hoc networks (VANETs), where timely message delivery and reliable transmission are of significant importance. We present an exact message delivery delay distribution for a two-lane road, where vehicles in one direction act as message carriers for the ones in the other direction and have the freedom to leave the road from randomly distributed exits with a certain probability. Our analysis offers a tool for an intelligent transportation system (ITS) service provider to determine the minimal separation between two consecutive RSUs for meeting a probabilistic requirement of the message delay. Simulation results show the accuracy of our analysis.
\end{abstract}

Keywords - Delay, vehicular ad hoc network, vehicle-toinfrastructure, disrupted connectivity, road side unit placement.

\section{INTRODUCTION}

Intelligent transportation systems provide substantial benefits to transport infrastructure. For instance, they can be used to avoid roadway congestion, which adversely affects travel times and fuel consumption. They also can monitor air pollution levels and collect information about driving habits. Currently, with the ubiquity of wireless communication devices in vehicles, new services such as providing access to email, news, and entertainment applications start to emerge.

However, the deployment of a fully infrastructure-based ITS is known to be very costly [1]. Indeed, it is very difficult to cover rural areas with sensors and RSUs such that every vehicle can always be connected to at least one nearby RSU during its trip within an area. Consequently, using vehicle-tovehicle communication is crucial to facilitate data reporting to RSUs, as they represent the gateways to the Internet and/or the rest of the ITS infrastructure. Therefore, vehicular ad hoc networks (VANETs) recently have started to attract attention from many researchers in both industry and academia [2][10]. The US Federal Communications Commission (FCC) has allocated $5.850-5.925 \mathrm{GHz}$ band to promote vehicular communications for safe and efficient highways. This band is planned to be used in the emerging radio standard for Dedicated Short-Range Communications (DSRC) [3] that supports roadside-to-vehicle and inter-vehicle communications.

$\S$ Dept. of Elec. Eng., United Arab Emirates University, Al-Ain, Abu Dhabi, UAE, 17555.

$\dagger$ †ept. of Elec. \& Comp. Eng., University of Toronto, Toronto, Ontario, Canada M5S 3G4.

$\ddagger$ Dept. of Elec. \& Comp. Eng., University of Waterloo, Waterloo, Ontario, Canada N2L 3G1.
For vehicular communication networks to become a reality, a number of technical challenges have to be addressed. First, it is difficult to maintain an end-to-end connection between vehicles and an RSU, while the vehicles are moving at high speeds, especially on roads with a low vehicle density. Second, data packets may suffer excessive delays when delivered to RSUs if they are carried by their originator vehicles to the nearest RSU. However, vehicle-to-vehicle communication can shorten the packet delivery delay if vehicles moving in the opposite direction are used. At the same time, vehicle-tovehicle communication with vehicles moving in the opposite direction may not be reliable as those vehicles may exit the road to uncovered areas before reaching any RSU.

Our objective in this paper is to analyze packet delivery delay with a reliable packet transfer for vehicle-to-infrastructure communications in a sparse vehicular ad hoc network (i.e., all data packets are to be delivered to RSUs). Our analysis aims at characterizing the packet delivery delay distribution for a two-lane road. Vehicles in one lane of this road are packet generators moving in a certain direction while vehicles in the other lane are packet carriers moving in the opposite direction and carrying the packet to the nearest RSU. Our analysis considers a simple, but reliable, packet delivery scheme where two replicas of each packet are generated. One replica is submitted to a packet carrier while the other is held by the packet generator until it meets an RSU. The packet delay distribution offers a design tool that can determine the maximum separation distance between two RSUs or the minimum number of RSUs covering a road segment for satisfying a probabilistic requirement of packet delivery delay.

\section{RELATED WORKS}

In the literature, most research works related to disrupted connectivity in vehicular ad hoc networks focus on connectivity analysis [4]-[6] and average message delay evaluation [5] [7]. Wu et al. in [8] present analytical models to study spatial propagation of information for one and two-lane straight roads. They focus mainly on information propagation speed for vehicle-to-vehicle communications. In [9], the authors analyze the probability of connectivity using RSUs. The average length of a connected path from any given vehicle to an RSU is also calculated. However, no study about packet delivery delay is provided. The feasibility of information dissemination using stationary supporting units (SSUs) is investigated in [10] mainly by computer simulations. However, vehicle-to-RSU packet delivery delay is not addressed.

To the best of our knowledge, no other study in the literature characterizes the delay and reliability of vehicle-to- 
infrastructure packet delivery using vehicle-to-vehicle communication.

\section{SYSTEM MODEL}

Consider a two-lane road. Each lane is a straight line with some fixed length $a$ meters and has two RSUs at its ends. Initially, vehicles are distributed on the road following a Poisson point process.

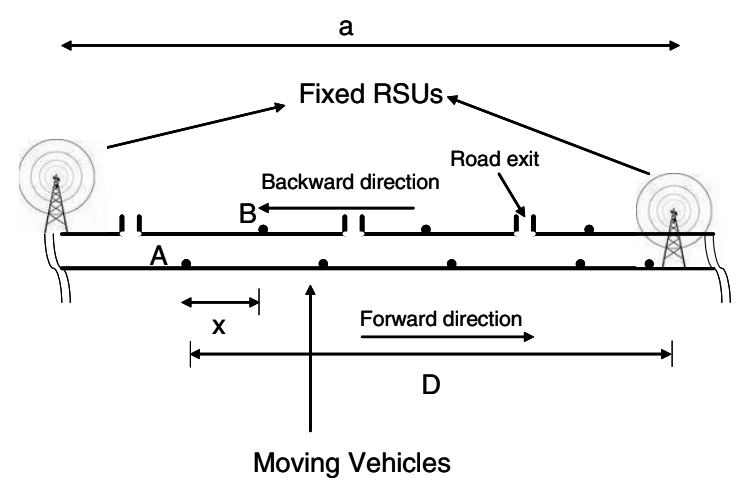

Fig. 1. Problem Description.

We are interested in a network scenario on highways or rural areas, where the vehicle density (defined as the average number of vehicles per unit road length) is low enough to have disrupted vehicle-to-vehicle and vehicle-to-RSU connectivity. With a high vehicle density, a multihop end-to-end path can be found between a vehicle and an RSU with a high probability; however, this case is out of the scope of this research.

Vehicles over the two lanes are moving in opposite directions as in Figure 1. Assume that all vehicles in the first lane are moving in the forward direction with a constant speed $V_{f}$. Conversely, in the other lane, vehicles move in the backward direction with another speed $V_{b}$. Therefore, the meeting opportunity happens only between vehicles moving in the opposite directions. Consider a straight road with exits or cross roads distributed randomly on the second lane as depicted in Figure 1. Consider that data packets are originated only from vehicles in the first lane such as vehicle $A$. Some vehicles may leave and others may join at any exit point along the road segment.

Since some vehicles may leave the road segment without delivering the packets they have received, we assume that each generator vehicle, such as vehicle $A$ in Figure 1, generates two duplicates of each of its packets. The first duplicate is held until the vehicle meets the first RSU on its way and the second is sent to the first approaching vehicle (vehicle $B$ in Figure 1) in the opposite direction. This approach is to increase the reliability of message delivery, which is required for data messages containing critical information.

The number of vehicles on each lane is Poisson distributed with vehicle density for the first lane $N_{1}$ and the second lane $N_{2}$. The vehicle densities $N_{1}$ and $N_{2}$ are assumed to be constant, i.e. over an observation period the average number of vehicles that leave the road segment under consideration is the same as the average number of vehicles that enter it.

As our objective in this paper is to analyze message delivery delay with reliable transmission in mind, we abstract the packet transmission process for simplicity. We assume all messages held by a vehicle will be transmitted during a meeting opportunity with a carrier vehicle with no bandwidth constraint or wireless transmission impairments. This assumption is also adopted by other researchers as in [8].

\section{Problem Formulation and Modeling}

\section{A. Problem Description}

The problem can be described by the aid of Figure 1. Our main objective is to find the maximum value of $a$ that allows a certain required vehicle-to-RSU message delivery delay to be attained for the majority of vehicle packets (i.e., $a$ should be selected to allow only a small fraction of packets to be delayed when arriving at an RSU). Indeed, timely message transfer is vital for information exchange over any ITS system as the information content may expire if arrived after a very large delay. Meanwhile, the number of RSUs that cover the road segment under study for a probabilistic delay requirement is directly related to $a$.

Furthermore, we aim at guaranteeing reliable packet transmission by allowing every packet generator vehicle to store a replica of its packets for direct transmission to an RSU when there is a meeting opportunity between them. Indeed, since every packet has two duplicates, late duplicates will be discarded (in a case both duplicates are successfully received).

In short, our problem is to find the minimum number of RSUs that can cover a road segment such that the required vehicle-to-RSU packet delivery delay is probabilistically satisfied for the first arrived duplicate. Note that packet carrier vehicles can exit the road with some probability from randomly distributed exits.

\section{B. Mathematical Model}

Consider the road segment depicted in Figure 1. A vehicle can leave the road segment from a cross road or a ramp with a probability $p_{c}$. We assume that the number of cross roads within the road segment follows a Poisson distribution with a parameter $\lambda_{c}$. Therefore, the vehicle will leave the road segment within a distance of $y$ with probability $p_{l}(y)$

$$
p_{l}(y)=1-e^{-\lambda_{c} p_{c} y} .
$$

Our target is to obtain an exact expression for the packet delivery delay cumulative distribution function $(\mathrm{CDF}) F_{T}(\cdot)$ in terms of $a$, taking into account that two replicas of every packet are submitted but only one is required to be delivered on time. The strategy has two benefits: First, it helps us to study how the delay distribution is affected by varying system parameters such as vehicle speeds $\left(V_{b}, V_{f}\right)$, vehicle densities $\left(N_{1}, N_{2}\right), p_{c}$, and $\lambda_{c}$; Second, it can be used to find the maximum value of the design parameter $a$ (or the RSU density 
$\frac{1}{a}$ ) that satisfies certain delay constraint $T_{\max }$ with a violation probability of at most $\epsilon$, as indicated in the following equation

$$
\begin{aligned}
& \text { maximize } \quad a \\
& \text { s.t. } \quad 1-F_{T}\left(T_{\max }\right) \leq \epsilon .
\end{aligned}
$$

The problem can be described analytically by

$$
\operatorname{Pr}(T \leq t)=\operatorname{Pr}(T \leq t, I=1)+\operatorname{Pr}(T \leq t, I=0)
$$

where $T$ is a random variable that denotes the packet delivery delay and $I$ is an indicator random variable denoting the status of the packet delivery by a carrier vehicle to an RSU. In the first case $(I=1)$, a carrier vehicle reaches the nearest RSU on its way carrying the packet under study. The second case $(I=0)$ indicates that the carrier vehicle exits the road before meeting an RSU.

First, we want to find the conditional CDF of the packet delivery delay given $I=1$ (i.e., the first term in the right hand side of (3))

$$
\operatorname{Pr}(T \leq t, I=1)=\operatorname{Pr}(T \leq t \mid I=1) \operatorname{Pr}(I=1) .
$$

Therefore, we condition on the location of the packet generator vehicle and the distance between the packet generator vehicle and the nearest packet carrier vehicle as in the following

$$
\begin{aligned}
\operatorname{Pr}(T \leq t, I=1)= & \int_{0}^{\infty} \int_{0}^{a} \operatorname{Pr}(T \leq t \mid I=1, D=d, X=x) \\
& \times \operatorname{Pr}(I=1 \mid D=d, X=x) f(d, x) d d d x
\end{aligned}
$$

where $D$ is a random variable representing the distance between the packet generator vehicle, $A$, (at the time when the packet is being sent) and the RSU as in Figure 1, $X$ is a random variable that characterizes the distance between vehicle $A$ and $B$, and $f(d, x)$ denotes the joint probability density function (PDF) of $D$ and $X$ and is given by

$$
f(d, x)=\frac{1}{a}\left(N_{1}+N_{2}\right) e^{-\left(N_{1}+N_{2}\right) x}, 0<d<a, x>0 .
$$

Note that the random variable $D$ is uniformly distributed since the vehicle location is uniformly distributed over $a$. In addition, a packet can be sent randomly at any time. The random variable $X$ is exponentially distributed since the vehicles form a Poisson point process.

For the case $I=0$, a similar equation to (5) holds (by replacing $I=1$ with $I=0$ in (5)).

The first term inside the integration of (5) is described by

$$
\begin{aligned}
\operatorname{Pr}(T \leq t \mid I=1, D= & d, X=x)= \\
& u\left(t-\min \left(\frac{a-d+x}{V_{b}}, \frac{d}{V_{f}}\right)\right)
\end{aligned}
$$

where $u($.$) is the Heaviside unit step function, which is used as$ a result of conditioning the packet delivery delay CDF on all the random variables involved. The minimum function reflects that the packet delivery delay is the minimum delivery time between two replicas in a case both of them reach RSUs. The replica carried by the generator vehicle arrives after a time of $\frac{d}{V_{f}}$, while the other replica arrives after $\frac{a-d+x}{V_{b}}$ if the carrier vehicle does no exit the road. Thus, the unit step function term can be expressed as

$$
\begin{aligned}
& u\left(t-\min \left(\frac{a-d+x}{V_{b}}, \frac{d}{V_{f}}\right)\right) \\
& \quad=\left\{\begin{array}{l}
u\left(t-\frac{d}{V_{f}}\right), \quad 0 \leq d \leq \min \left(\frac{(a+x) V_{f}}{V_{b}+V_{f}}, a\right) \\
u\left(t-\frac{a-d+x}{V_{b}}\right), \quad \min \left(\frac{(a+x) V_{f}}{V_{b}+V_{f}}, a\right) \leq d \leq a .
\end{array}\right.
\end{aligned}
$$

The probability that the packet carrier vehicle will not leave the road before delivering its packets to an $\operatorname{RSU}(I=1)$, given that the carrier vehicle is located at a distance of $a-d+x$ from the nearest RSU on its way, can be obtained as

$$
\operatorname{Pr}(I=1 \mid D=d, X=x)=1-p_{l}(a-d+x) .
$$

On the other hand, when a packet carrier vehicle exits the road, the packet replicas it carries are considered lost. The probability of this case can be expressed as

$$
\operatorname{Pr}(T \leq t \mid I=0, D=d, X=x)=u\left(t-\frac{d}{V_{f}}\right) .
$$

Again, as we condition the packet delivery delay CDF on all the random variables involved, we use the unit step function to determine the conditional packet delivery delay CDF. The probability that a carrier vehicle will leave the road given that it is located at a distance of $a-d+x$ from the nearest RSU can be obtained as

$$
\operatorname{Pr}(I=0 \mid D=d, X=x)=p_{l}(a-d+x) .
$$

Finally, we obtain the exact expressions for the packet delivery delay CDF as presented in (12) by using (3)-(11).

\section{Simulation Results And Discussion}

In this section, we first validate our analysis using computer simulations. Subsequently, we use our analytical model to study the effect of changing system parameters on the required RSU density, under the constraint of satisfying the packet delivery delay requirement probabilistically.

\section{A. Model Validation}

The ns-2 simulator is used in order to validate our analysis. We implement the mobility model mentioned in Section III inside the ns-2 mobility scenario generator. In this mobility model, vehicles are forced to move with a constant speed over a two-lane straight line along a road segment of fixed length. One RSU is placed at each end of the road segment. Vehicles in the first lane are moving in the forward direction, while in the second lane vehicles are moving in the opposite direction as in Figure 1. Only vehicles on the first lane can be active traffic sources, while vehicles in the other lane act as packet carriers. The IEEE 802.11 is used as the medium access control (MAC) protocol for the single channel system in the simulator. Data traffic is generated by constant bit rate sources with very low rate compared to the channel data rate, which guarantees that the contact time between two vehicles is sufficient for them to exchange one packet. Table I gives 


$$
\begin{aligned}
& \operatorname{Pr}(T \leq t)=\left[\left(\frac{V_{f}}{a}\right) t+K\left(1-e^{-t V_{b}\left(\lambda_{c} p_{c}+N\right)}\right)+\frac{e^{-t V_{b}\left(\lambda_{c} p_{c}+N\right)}}{a \lambda_{c} p_{c}}-\frac{e^{-t \lambda_{c} p_{c} V_{b}}}{a \lambda_{c} p_{c}}\right] u(t) \\
& +\left[\frac{e^{-t V_{b} \lambda_{c} p_{c}}}{a \lambda_{c} p_{c}}-K\left(e^{-\lambda_{c} p_{c} a} e^{t \lambda_{c} p_{c} V_{f}}-e^{-t V_{b} \lambda_{c} p_{c}} e^{-N V\left(t-\frac{a}{V}\right)}\right)-\frac{e^{-t \lambda_{c} p_{c} V_{b}} e^{-N V\left(t-\frac{a}{V}\right)}}{a \lambda_{c} p_{c}}\right] u\left(t-\frac{a}{V}\right) \\
& +\left[\begin{array}{l}
1-\left(\frac{V_{f}}{a}\right) t-K e^{-t V_{b} \lambda_{c} p_{c}} e^{-N V\left(t-\frac{a}{V}\right)}+\frac{e^{-t \lambda_{c} p_{c} V_{b}} e^{-N V\left(t-\frac{a}{V}\right)}}{a \lambda_{c} p_{c}}-\frac{e^{-t V_{b}\left(\lambda_{c} p_{c}+N\right)}}{a \lambda_{c} p_{c}} \\
-K\left(1-e^{-t V_{b}\left(\lambda_{c} p_{c}+N\right)}\right)+K e^{-\lambda_{c} p_{c} a} e^{t \lambda_{c} p_{c} V_{f}}
\end{array}\right] u\left(t-\frac{a}{V_{f}}\right) \\
& V=V_{b}+V_{f}, N=N_{1}+N_{2}, K=\frac{N}{a p_{c} \lambda_{c}\left(\lambda_{c} p_{c}+N\right)}
\end{aligned}
$$

the key system parameter values used in the analysis and simulations unless otherwise specified.

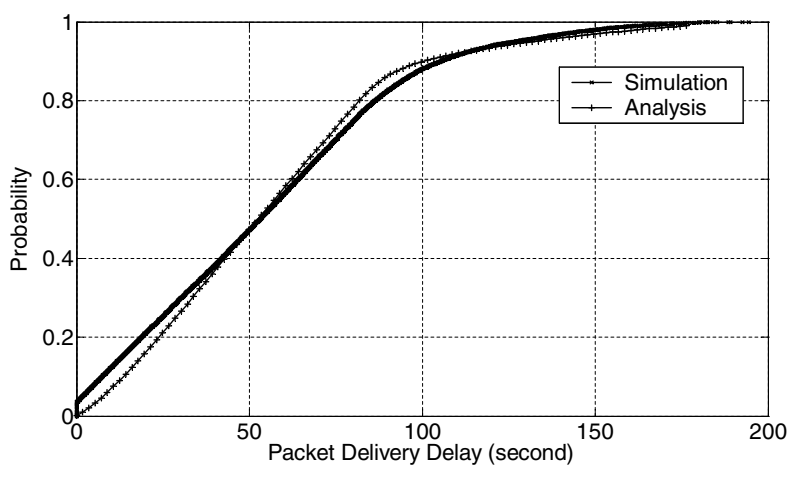

Fig. 2. Packet delivery delay CDF (analysis and simulation).

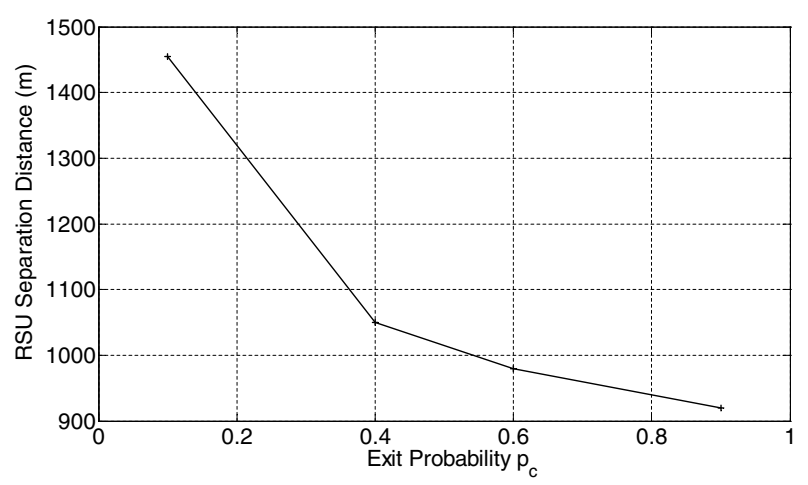

Fig. 3. RSU separation distance with different exit probabilities.

Figure 2 shows a histogram of the packet delay probability distribution (with the delay values approximated to the nearest integer) obtained from the simulator and the analytical results. The simulated histogram is obtained using 600 runs, where each run represents $200 \mathrm{~s}$ of system time. Figure 2 demonstrates that there is a close match between the analysis and the simulation results, indicating that our analysis is accurate in characterizing the packet delivery delay distribution.

\section{B. RSU Density and System Parameters}

Here, we study the effect of changing key system parameters such as exit probability, exit density, vehicle density, and speed difference (between the vehicles moving in the forward and the backward directions) on the required RSU density. Indeed, the
TABLE I

SYSTEM PARAMETERS

\begin{tabular}{|c|c|}
\hline System Parameter & Value \\
\hline \hline Road Segment Length $(a)$ & $5 \mathrm{~km}$ \\
\hline$N_{1}$ & $0.005 \mathrm{~m}^{-1}$ \\
\hline$N_{2}$ & $0.005 \mathrm{~m}^{-1}$ \\
\hline Exit Probability $p_{c}$ & 0.3 \\
\hline Exit Density $\lambda_{c}$ & 0.002 \\
\hline$V_{f}$ & $25 \mathrm{~m} / \mathrm{s}$ \\
\hline$V_{b}$ & $30 \mathrm{~m} / \mathrm{s}$ \\
\hline
\end{tabular}

separation distance $a$ between two consecutive RSUs translates directly to the RSU density. Here, we solve (2) to get the value of $a$, which satisfies a $T_{\max }=30 \mathrm{~s}$ and $\epsilon=5 \%$ for different values of key system parameters.

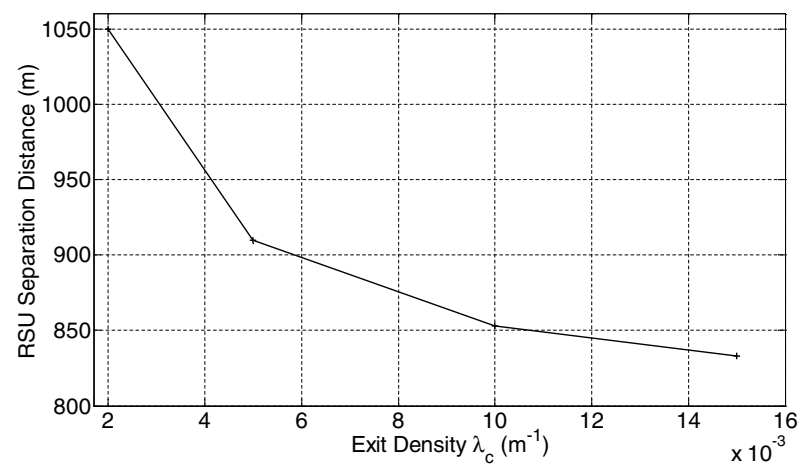

Fig. 4. RSU separation distance with different exit densities.

Figure 3 illustrates the effect of changing the exit probability on the RSU density. The figure indicates that, as the exit probability increases, the RSU separation distance satisfying $T_{\max }$ and $\epsilon$ decreases significantly. This is expected as, when carrier vehicles have more tendency to leave the road, the more likely that a packet will reach an RSU carried only by its generator. Certainly, a high leaving probability increases the number of RSUs required to cover a road for certain packet delivery delay requirements.

The relation between different exit densities and the RSU separation distance is depicted in Figure 4. The figure shows that the required RSU density decreases when the density of exits decreases. This is anticipated as for the same road segment, when we decrease the available number of exits, the number of carrier vehicles that exit the road decreases (for 


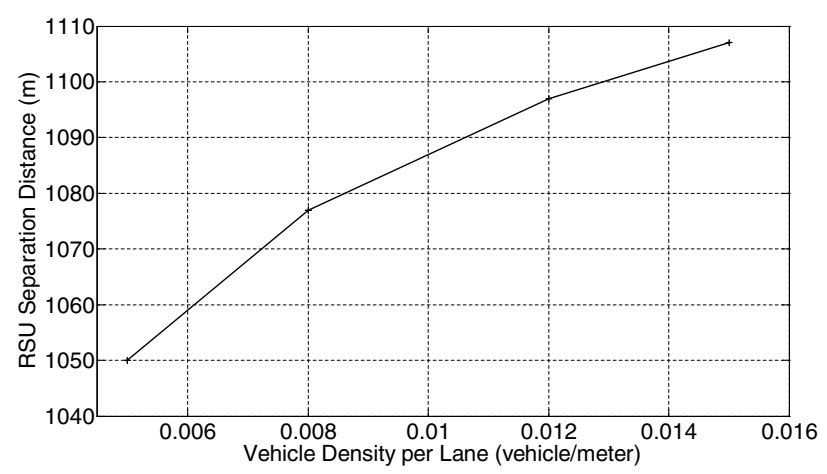

Fig. 5. RSU separation distance with different vehicle densities.

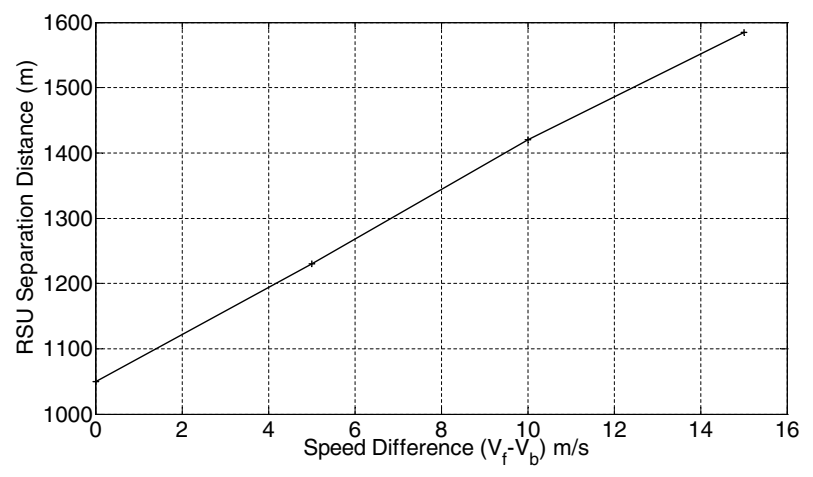

Fig. 6. RSU separation distance with $\left(V_{f}-V_{b}\right)$

the same exit probability $p_{c}$ ). This implies an increasing role of packet carrier vehicles in delivering packets to an RSU, which translates directly to a higher probability of small packet delivery delay (or higher $a$ for the same $T_{\max }$ and $\epsilon$ ).

Figure 5 shows the variation of the RSU separation distance with different vehicle densities, under the assumption $N_{1}=N_{2}$. It can be clearly seen from Figure 5 that, as the vehicle density increases, the RSU separation distance increases. The reason is that a low vehicle density decreases meeting opportunities between generator and carrier vehicles for a certain $a$, which gives less favor to packet transfer on the backward direction, and in turn increases the probability of a long delay.

Figure 6 shows how the RSU separation distance varies when the speed difference $V_{f}-V_{b}$ changes. The figure shows that the RSU separation distance increases with the speed difference $V_{f}-V_{b}$. Generally, increasing $V_{f}$ leads the packet delivery delay to decrease since generator vehicles can reach an RSU faster.

\section{Discussion}

The reliable packet delivery scheme we introduce in this paper depends mainly on the ability of the packet generator vehicle to store its packets until it meets an RSU on its way. We control the separation distance between consecutive RSUs (or RSU density) to limit the packet delivery delay probabilistically. However, if a non-reliable scheme is used (e.g., a generator vehicle sends its packets to a carrier vehicle without storing a replica), another limitation is imposed on the RSU density, which is the packet loss probability (PLP) as the following equation implies

$$
P L P=1-\frac{1-e^{-p_{c} \lambda_{c} a}}{p_{c} \lambda_{c} a} .
$$

We can infer from (13) that, as we increase $a$, the packet loss probability increases. This means that a small $a$ (or a large RSU density) is required to achieve a small, and hence acceptable, packet loss probability.

Given the recent advances in the capacity of memory chips, vehicles are able to store a fairly large amount of packets in their buffers before meeting an RSU. Therefore, storing one replica of every packet in the generator vehicle does not represent a disadvantage for our scheme in comparison with a non-reliable scheme. In addition, our scheme leads to a smaller RSU density compared to that for a non-reliable one.

\section{CONCLUSION}

In this paper, we present a study of the relation between packet delivery delay and RSU density for vehicular-to-RSU communication in sparse vehicular ad hoc networks. We investigate the case where vehicles moving in one direction send their packets to vehicles traveling in the opposite direction in order to deliver the packets to the nearest RSU. We consider a simple, but reliable scheme, which attempts to send two replicas of every packet to RSUs. One replica is carried by its originator on its way to the nearest RSU, and the other is carried by a vehicle moving in the opposite direction. An exact packet delivery delay distribution is derived. Simulation results validate the accuracy of our analysis.

\section{REFERENCES}

[1] Ten-Year National Program Plan and Research Agenda for Intelligent Transportation Systems in the United States, ITS Amer., U.S. Dept. Transp., Washington, DC, Oct. 2001.

[2] A. Abdrabou and W. Zhuang, "On a stochastic delay bound for disrupted vehicle-to-infrastructure communication with random traffic," Proc. IEEE Globecom'09, pp. 3904-3909, Dec. 2009.

[3] IEEE. 5.9 GHz Dedicated Short Range Communications (DSRC), [online]. Available: http://grouper.ieee.org/groups/scc32/dsrc/.

[4] M. Khabazian and M. Ali, "A performance modeling of connectivity in vehicular ad hoc networks," IEEE Trans. Veh. Tech., vol. 57, no. 4, pp. 2440-2450, Jul. 2008.

[5] A. Agarwal, D. Starobinski, and T. Little, "Analytical model for message propagation in delay tolerant vehicular ad hoc networks," Proc. IEEE VTC08, pp. 3067-3071, May 2008.

[6] S. Panichpapiboon and W. Pattara-atikom, "Connectivity requirements for self-organizing traffic information," IEEE Trans. Veh. Tech., vol 57, no. 6, Nov. 2008, pp. 3333-3340.

[7] R. Groenevelt, P. Nain, and G. Koole, "The message delay in mobile ad hoc networks," ACM Perform. Eval., vol. 62, no. 1-4, pp. 210-228, May 2005.

[8] H. Wu, R.M. Fujimoto, G.F. Riley, and M. Hunter, "Spatial propagation of information in vehicular networks," IEEE Trans. Veh. Tech., vol. 58, Jan. 2009, pp. 420-431.

[9] S. Yousefi, E. Altman, R. Elazouzi, and M. Fathy, "Improving connectivity in vehicular ad hoc networks: An analytical study," Computer Communications, vol. 31, 2008, pp. 1653-1659.

[10] C. Lochert, B. Scheuermann, M. Caliskan, and M. Mauve, "The feasibility of information dissemination in vehicular ad-hoc networks," Proc. WONSO7, Jan. 2007, pp. 92-99. 